\title{
Rotator Cuff Injury, CTCAE
}

National Cancer Institute

\section{Source}

National Cancer Institute. Rotator Cuff Injury, CT CAE. NCI Thesaurus. Code C143227.

A disorder characterized by an injury of the rotator cuff. 